\title{
El Sur de Africa y el fin de la guerra fría
}

\begin{abstract}
$R$ I С $А R D C$ С $T$ fuc investigador del Royzl Institute of International Affairs en Londres, y editorialista del matutino londinense, The Guardian. Es ahora profesor-investigador de asuntos de Africa y el muncio irabe en el Instituto de. Estudios Internacionales de la Universidad de Chile. Es el coautor de The Appeasers (Weidenfeld and Nicolson, 1963) y el director de ESTUDIOS INTERNiclosilles. Este artículo esti escrito en base a una conferencia dictada en el Salón de Honor de la Universidad de Chile, el 27 de octubre de 1966.
\end{abstract}

Pocos problemas internacionales han permanecido an largo tiempo sin solución como los de Sudáfrica. Hace exactamente 20 años que el Gobierno de la India advirtió, por primera vez, que la política interna de Sudáfrica era un asunto que concernia a la política internacional. En junio de $19 \pm 6$, la India solicitó ante las Naciones Unidas, que el problema del trato dado a la colonia hindú residente en Sudáfrica fuera incluido en la agenda prorisoria para la segunda parte de la primera reunión de la Asamblea General de las Naciones Unidas. Pero aquello que comenzara siendo solamente una protesta individual en contra de una nación por negarse esta última a conceder los mais elementales derechos humanos a su pequeña comunidad hindú, creció como una bola de nieve, transformándose en una amplia crítica internacional, cuando Sudáfrica se anexó de facto los mandatos vecinos de África Sudoccidental. El gobierno sudafricano rechazó la recomendación hecha por la Asamblea General para que el territorio fuera gobernado por el sistema de Fideicomiso Internacional. Y, finalmente, en 1952, el problema mismo del apartheid fue tratado en Ias Naciones Unidas. Trece estados afroasiáticos solicitaron que el problema de los conflictos raciales en Sudífrica, suscitado por la política de apartheid de ese gobierno, fuese incluido en la agenda de la séptima reunión de la Asamblea General.

Desde aquella fecha, en nuestra opinion, Sudáfrica ha sido virtualmente denigrada y execrada por los gobiernos de todos los paises del mundo. Bástenos citar aqui, de una de las mis recientes condenas, parte del comunicado emitido el lt de septiembre de 1966 en Londres, al térnino de la Conferencia de Primeros Ministros de la Comunidad Británica de Naciones: 
E S T U D I O S I X TER X A C I O $X$ A L E S

"Los Primeros Ministros declaran que ningún sistema politico basado en la discriminación racial puede ser tolerndo, ya que esto constituyc un atentado contra la libertad tanto de aquéllos que la imponen como de aqueilos que la soportan. Consideran que las condiciones discriminatorias, tanto de carácter político como social, económico y cducativo, impucstas por una minoria sobre la mayoria, en beneficio de unos pocos privilegiados, constituyen una descarada violación de los más fundamentales principios de los derechos humanos"1.

No obstante, a pesar de los buenos propósitos y de las resoluciones adoptadas por las Naciones Unidas, Sudáfrica, hasta el momento, permanece segura -reprobada por la opinión internacional, pero aparentemente a salvo de toda acción internacional.

En la práctica, aunque los gobiernos de los estados soberanos suscriban ciertos principios morales, por lo general todas sus acciones están gobernadas lamentablemente por lo que ellos piensan que constituye su propio interés político o económico. Nuestra intención es explicar aqui por qué los esfuerzos internacionales por derrocar la supremacia blanca en Sudáfrica han fracasado, a pesar de que la politica de apartheid de la minoria racista blanca haya sido universalmente condenada. Trataremos, al mismo tiempo, de observar con cierta perspectiva, algunos de Ios más recientes acontecimientos que han dado motivo, en general, a una renovada atención sobre el problema de África del Sur.

Primeramente, hemos sido testigos, durante los meses recién pasados, del asesinato del Primer Ministro sudafricano, Dr. Verwoerd. Para asombro de todos, fue ultimado, no por alguna victima negra de su politica de apartheid, sino, paradójicamente, por un así llamado "blanco pobre". Los sudafricanos han preferido declararlo insano pero, al parecer, en un momento de lucidex, declaró que consideraba que el Dr. Verwoerd invertia demasiado dinero público en proyectos destinados a beneficiar a la mayoria negra. En el mundo enrevesado de la politica sudafricana donde, según el actual Primer Ministro, liberalismo y comunismo son equivalentes, resultaba inevitable, tal vez, que el archiapóstol y más fantítico defensor del apartheid -y, de hecho, su principal filósofo- parecicse un "moderado" cuando sobrevino su mucrte. En verdad, comparado con șu sucesor, Mr Vorster, quien, como Mlinistro de Justicia, fue el responsable de algunas de las más odiosins medidas que se hayan registrado, el Dr. Verwoerd resultaba un modelo de marnanimidad $y$ esclarecimicnto. Durante la Segunda Guerra Mundial, por ejemplo, Verwoerd fuc solamente el editor del principal periodico pro-nazi, mientras que Afr. Vorster fue encarcelidio por sus actividades pro-nazis.

El segundo acontecimiento que tiene relación con el problema sudafricano

"Guardian (Alasebestet), 13-sept., 155 .

$$
[96 \cdots]
$$


es la curiosa decisión adoptada en julio de 1966 por la Corte Internacional de Justicia de La Haya, respecto de la administración de Africa Sudoccidental. Como recientcmente las Naciones Unicies volaron una resolución concerniente al africa Sudoccidental, y por lango tiempo esta región ha sido considerada como la clave del problema sudafricano, debemos exponer aquí algunos detalles.

El territorio dui Africa Sudoccidental, perteneciente a Alemania, fue ocupado por los sudafricanos a comienzos de la Primera Guerra Mundial. Terminada la guerra, las potencias vencedoras, en la Conferencia de Paz de Versailles, rechazaron a Sudáfrica el derecho de conquista, y decidieron que el África Sudoccidental, asi como todos los restantes territorios coloniales conquistados a Alemania, debían quedar bajo el mandato de la Liga de las Naciones. El artículo 22 del Tratado de Versailles estipulaba que como el territorio del África Sudoccidental estaba "todavía habitado por pueblos incapaces de gobernarse a si mismos bajo las agobiantes condiciones del mundo moderno" debería aplicarse a ellos el principio de que el bienestar y desarrollo de tales pueblos, constituye una misión sagrada para la civilización.

Esta misma frase surge una y otra vez en cualquier discusión sobre el problema del África Sudoccidental. En 19́20, la comunidad internacional, mediante la Sociedad de Naciones y más tarde a través de las Naciones Unidas, concordaba siempre en que el futuro bienestar del medio millón de nativos del África Sudoccidental, quienes habian sido miserablemente tratados durante el período colonial alemán, debería ser considerado como "una misión sagrada para la civilización". Desde entonces, la comunidąd internacional ha tratado de persuadir a Sudífrica a proceder de acuerdo con este noble principio. Pero sin éxito. La prolongada campaña de las Naciones Unidas contra la administración sudafricana del territorio de Africa Sudoccidental, se ha visto virtualmente detenida desde noviembre de 1960, cuando Etiopía y Liberia, en representación del grupo africano de Jas Naciones Unidas y en su calidad de paises africanos negros que habian sido miembros de la Sociedad de Naciones, encomendaron la totalidad de este problema a la Corte Internacional. Ambos paises pidieron a la Corte declarar que Sudátrica practicaba el apartheid en el África Sudoccidental y que, al actuar de esa manera, se violaban los términos del mandato. Pidicron, además, que esta acción cesara de inmediato. Dos años más tarde, la Corte determinó, por el escaso margen de 8 votos contra 7, que existian antecedentes jurídicos para emitir un fallo sobre la validez del caso. Sin embargo, los dos jueces de la Comunidad Británica de Naciones opusicron a csto una fuerte resistencia, alegando que las causas no cran "suficientes como para justificar una determinación legal objetiva", y que se habia pedido a la Corte "asumir lat responsabilidad de una tarea que, en un análisis final, dificilmente podría ser considerado como de carácter judicial". 


\section{E 5 TLDIOS INTER NACIONALES}

En 1962 esta oposición no logró tener exito. Pero $t$ años mis tarde, en julo de 1966 , con motivo del cambio de dos jucecs, por mucrte de uno y retiro del otro, la decisión fue resocada mediante el voto decisivo del presidente, el juez australiano Sir Percy Spender, y la Corte decidió que Liberia y Eciopía carecian de poderes legales en el caso y la solicitud, por lo tanto, fue recinazada. El juez norteamericano Jessop, vale la pena recordarlo, emitió un juicio de enérgica reprobación, diciendo que lamentaba profundamente el verse obligado a dejar constancia que la determinación de la Corte carecia por completo de fundamento legal. Includablemente, el efecto a largo plazo de la decisión habría de minar la confianza en la capacidad judicial de la Corte Internacional y constituiría una grave desilusión para aquéllos que anhelaban una determinación basada sobre la aceptación universal del Derecho Internacional. Hace algunos meses, los estados africanos representados en las Naciones Unidas y la uRss lograron impedir un aumento del presupuesto destinado a la Corte Internacional de Justicia, como una represalia por la decisión sobre Africa Sudoccidental'2. Pero el resultado inmedia to ha sido traer nuevamente el problema de Africa Sudoccidental a la arena politica que, por supuesto, ha sido discutido en la última sesión de la Asamblea General. Los estados africanos se han mostrado, como es natural, reacios a emprender ninguna gestión por conducto de la Corte Internacional. El particlo político domin nte, Unión Africana de Kenya, anunció después de conocer el veredicto de la zorte, que ha llegado el momento de que los pueblos del Africa Sudoccidental se levanten y utilicen todos los medios posibles y apropiados para desterrar la opresión racial y colonial. Ha llegado el tiempo -expresó- de formar una fuerza armada capaz de derrocar los regimenes de Sudúfrica ${ }^{3}$. Así, la decisión de la Corte Internacional de Justicia unida al asesinato del Dr. Verwoerd, han introducido nuevos elementos en el puzzle sudafricano. A estos elementos podría también agregarse la independencia, otorgada hace pocos meses por Gran Bretaña, a los pequeños estados de Botswana y Lesotho; junto a la posición de los ejércitos cle liberación africana en las colonias portuguesas de Angola y Mozambique y, por supuesto, la aún irresuelta crisis de Rhodesia del Sur.

Se ha dicho que la historia de todas las sociedades humanas es la historia de las transformaciones y que si ellas no se hacen pacíficamente deberán realizarse violentamente. El problema africano que ha preocupado a las Nacio. nes Unidas durante los últimos veinte años, en especial descle que fuera aprobada la Declaración sobre Colonialismo en 1960, ha consistido en cómo proteger estos cambios - la liberación de la mayoria negra en los paíscs del África del Súr-sin encender al mismo tiempo un conflicto racial c'lyas consecuencias repercutirian más allá del Affrica misma.

Tres argumentos han siclo tradicionalmente empleados por aquellos que

"Coterdian, it, act. 1965.

stbid, 20 julio, l9c5. 


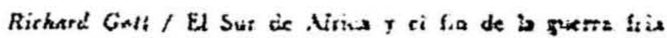

patrocinan una acción international contra el Srica del Sur. Primeramente, Suditrica ha violade sus compromisos hacia la Carta de las Naciones Lindas. Los artículos $5 j$ y 5 t; exigen que todos los estatos miembros le las Naciones Unidas deben cooperar con la Organizacion en promover "el respeto universal y la observancia de los derechos humanos y las fibcrades fundanentales para todos, sin distinción de raza, sexo, lenguaje o religión“. En segundo lug:ar, existe el inminente peligro de un conflicto racial de proporciones, en Sudúfrica y la acción para prevenirlo debe ser adoptada cuanto antes. La politica de apartheid sudafricana y el repetido incumplimiento de los acuerdos de las Naciones Unidas adoptados, como se expresa en varias resoluciones, constituye una "amenaza para la paz", lo que deja abierto el camino para que las Naciones Unidas adopten mediclas económicas y/o, militares conura Sudafrica, como lo estipula el capitulo vir de la Carta.

De hecho, hay quienes sostienen que la guerra racial ya ha comenzado en el mundo - sin esperar a que la chispa sudafricana encienda la hoguerat. Ciertamente, los actuales disturbios raciales en los Estados Unidos con el tan prolongado e ineritable rechazo blanco contra todos aquellos que aspiran a ir más allá del mero principio formal en sus derechos politicos, parecerian respaldar esta tesis. También existen otros ejemplos. Gran Bretaña, pais de actitud liberal por tradición hacia la inmigración, ha buscado protegerse del problema racial, cerrando sus puertas a todos, salvo un puñado; los inmigrantes de color. La Unión Soviética, a su vez, ha tenido sus problemas raciales, como lo demostraron en Moscú, en 1963, las manifestaciones en la Universidad Patricio Lumumba. Tampoco resulta demasiado atrevido considerar a la actual guerra de Vietnam, no como la última secuela de la guerra fría, sino como el primer choque de un nuevo conflicto racial en el mundo.

Es desde este ángulo, el de un inminente conflicto racial, como ha sido corrientemente considerado el problema del África del Sur. Existe, sin embargo, un tercer argumento que justificaria la acción. Siempre ha existido, en el Occidente especialmente, el temor de que si una acción internacional no es emprendida contra la supremacia blanca en África del Sur, los estados africanos podrian decidirse a resolver por si mismos el conflicto con Sudáfrica $y$, de paso, pedir la ayuda rusa o china. Desde el punto de rista de los países no-alineados, los estados independientes del África siempre pueden insinuar que podrian aliarse con los comunistas en un amplio canpo de posibilidades, a menos que el Occidente esté dispuesto a actuar contra f.frica del Sur. En el argumento de los nacionalistas africanos, como lo ha sugeridio una autoridad en esta materia, queda implicito que "si cl Occidente rehusa ayudar en la campaña de liberación, fucrzas hostiles a los interescs occidentales podrian perfectamente llegar a dominar la lucha y, de tener exito, producir trastornos

-Ver La obra The Race W'er de Ronsld Segal, Jonathan Cape, Londres. 


\section{ESTUDIOS I N TER N A C I O N A L E S}

politicos internos $y$ externos hacia dichos intereses Sc pretende, no obstante, demostrar aqui que de todas estas presiones, esta última es, por aliora, la menos efectiva, debido en gran medida, a los sorprendentes cambios ocurridos dentro y fuera de Africa recientcmente. De hecho, es necesario observar los problemas del Africa del Sur dentro del contexto de la nueva situación mundial creada por la terminación de la tradiçional guerra fría entre los Estados Unidos y la Unión Soviética y el consiguiente colapso del viejo concepto de "no-alineamiento" que en gran parte se basaba en la existencia de las hostilidades entre estos dos grandes poderes.

Evidentcmente, en la etapa presente, no se puede ser dogmático. Las guerras frías no se descongelan de un día para otro y en muchas áreas de roce aún perdurarán los conflictos. Por esta misma razón, los historiadores del futuro posiblemente dudarán en la atribución de una fecha exacta al término de la guerra fria, aunque para nosotros, hoy en dia, resulte claro que el convenio para la proscripción de las pruebas nucleares en 1963, señaló un desahogo, tres el cual tanto los Estados Unidos como la Unión Soviética manifestaror! un mutuo interés en no alterar el statu quo.

Sin embargo, en lo que a África respecta, así como para lo que se denominaba el Tercer Mundo, el aspecto significativo del término de la guerra fría lo constituye el virtual retiro de la Unión Soviética de una posición internacional avanzada $y$, lo que un escritor ha llamado "Ia dislocación del comunismo como fuerza revolucionaria mundial"'.

Para comprender la repercusión de este hecho sobre el mundo no-alineado, sería útil comparar el conflicto de la guerra fria con la actividad de dos magnetos igualmente poderosos que tratan desesperadamente de atraer a su propia esfera de influencia un trozo de metal. En tanto que los magnetos -Rusia y Norteamérica- tengan igual potencia, el trozo de metal, que representa al mundo no-alineado, permanecerá equidistante de cada uno de ellos. Pero si uno de los imanes pierde su poder de atracción, el trozo de metal, ineluctablemente será atraído por el otro. Es éste el fenómeno que nosotros hemos presenciado durante los últimos años, aunque solamente se nos haya hecho evidente durante los últimos dieciocho meses.

La creciente preocupación de la Unión Soviética por la fisura de sus rela, ciones con China, además de sus propios problemas políticos y económicos, juntamente con su desco de no repetir la experiencia cubana en otras partes del mundo, le ha hecho sentir aversión hacia la idea de mantener esa continua confrontación con los Estados Unidos. Aquel inconfortable equilibrio que existia entre las dos mayores potencias y que pernitia que floreciese el concepto del tercer mundo, ya no es válido. Como consecuencia práctia, las naciones más importantes del mundo no-alineado tales como Indonesia, Gui-

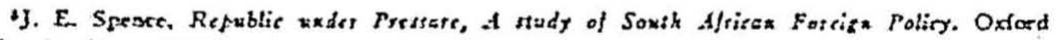
Linicruity Pres, 1965, p. H.

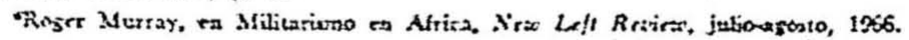


nea, Ghana, India y Brasil, se han acercado, en diferentes grados hacia la órbita occidental. $Y$ de los cinco dirigentes neutrales principales - Tito, Nasser, Nehru, Nkrumah y Sukarno-, quienes, por citar un ejemplo cie su posición noalincada, solicitaron a Eiscnhower y hinuschey que se reunieran durante las sesiones de las Naciones Unidas de 1960, solamente Tito y Nasser conservan aún la fachada de la politica de no-alineación.

En Africa, el efecto de esta tendencia ha sido devastador, particularmente en la lucha por la liberación del pueblo sudafricano. Pues en Africa esta desviación del énfasis de la no-alineación en un sentido favorable al Oeste, no ha sido confinada solamente a las naciones principales. Una serie de golpes de estado surgidos en los países más débiles y más pobres ha dado lugar a regímenes que refuerzan la tendencia al alineamiento de facto con el Occidente. $Y$ la inestabilidad resultante ha producido en la fragmentada politica continental el colapso de la corriente hacia el panafricanismo y una tendencia general en todos esos países a preocuparse de sus asuntos internos -o a empeorarlos- más bien que a tratar de generar una presión internacional contra el África del Sur. "La desarticulación de la estrategia del mundo comunista"z es ciertamente una de las razones de esta situación. Y no se nos puede acusar de maquiavelismo si advertimos la mano de los "servicios de inteligencia de las principales potencias" en algunos de los paises que recientemente han cambiado sus regímenes, aunque en la mayor parte de ellos, desde Glana y Nigeria a la República Centro Africana y Dahomey, las contradicciones de su política interna dan sobrados motivos para explicarla. Pero no nos compete explicar en detalle, en este momento, el porqué estos acontecimientos han ocurrido. Haremos constar, meramente, lo que ha sucedido para poder deducir conclusiones sobre la secuela de acontecimientos que como resultado de todo lo anterior, han venido produciéndose en el Africa del Sur.

La importancia de los recientes sucesos se explica mejor oponiéndoles la experiencia histórica de la última década. En estos últimos meses se cumplió el décimo aniversario de la crisis del canal de Suez - la invasión de Egipto por las fuerzas de Isracl, Gran Bretaña y Francia. En aquel tiempo, 1956, Africa estaba dominada militar y políticamente por Gran Brctaña y Francia. El movimiento de liberación en Argelia apenas había comenzado. Kwame Nkrumah, el líder político más importante de la Costa de Oro, aún no había guiado a su Partido hacia la independencia y el establecimiento del estado de Ghana. Jomo Kenyatta todavia estaba preso por su participación en el levantamiento Mau Mau en Kenya. El Dr. Hastings Banda, actualmente Primer Ministro de. Malawi, era en esa época un oscuro doctor que vivia en el extranjero y no había puesto los pies en su patria desde hacia 20 años. $Y$ más al sur, la dominación blanca se hallaba firmemente enclavada. Aparte de Sudafrica, el único estado importante libre del dominio colonial europeo cra el Egipto de Nasser.

TFrase de Roger Mluray. 
Paises mis pequeños - Liberia y Etiopia- habian logrado mantencr si independencia a través del poderio colonial, pero, fuera de cstas excepciones, Africa, hace sólo 10 años, era atun, de hecho, una colonia anglo-[rancesa.

Los habitantes blancos del extremo sur del $\mathcal{X}$ frica tenian pocos motivos para suponer que sobrevendria cambio alguno. Ciertamente, una descolonizacion gradual parecia probable en el África Occidental -una zona poco acogedora para el blanco, doncle nuuy pocos colonos europcos se habian establecido. Pero la minoriạ blanca dominante en Rhodesia del Sur, en las colonias portuguesas y en Sudáfrica misma, diffcilmente podia imaginar una situación en la que los cambios politicos llevaran al poder a la mayoria negra africana dentro de sus propios países o ni tan siquiera cerca de sus fronteras.

Pero después de 1956 las cosas cambiaron drásticamente. El Africa negra, con Ghana panafricanista a la cabeza, conquistó su independencia. Los británicos, bajo la administración conservadora, apresuraron este proceso y, Kenya y Tanganyika, tradicionalmente considerados como "países de blancos", quedaron bajo el dominio de la mayoría negra. Los franceses, bajo el gobierno de De Gaulle, juiciosamente abandonaron sus colonias. Los belgas abandonaron el Congo, y la Federación Centroafricana que había unido a Rhodesia del Nortc y del Sur con Nyasaland, se desintegró. Zambia y Malawi emergieron con su propia independencia. El África negra se fue moviendo hacia el sur y a medida que lo hacía, la protesta internacional contra la supremacía blanca en África del Sur fue subiendo de tono, y en aquellos paises en que las poblaciones blancas aún mantenian su predominio, éstas se volvieron cada vez más determinadas a aferrarse a lo que tenían.

Pero la aventura de Sucz, que en parte fuera responsable de haber iniciado esta rápida descolonización, tuvo un importante efecto psicológico sobre el Occidente. Porque al lograr Egipto infligir una derrota política de proporciones a los poderes de Europa Occidental, se creó la ilusión de que los estados africanos eran realmente más poderosos de lo que sus fuerzas militares o su cohesión política permitian imaginar. Los poderes europeos no poseían ni la fuerza militar ni la determinación politica para poder aplastar los movimientos anticolonialistas en África, y por lo tanto, resultaba fácil exagerar la decadencia de Occidente y el poder de los nuevos estados. Esto explica el que los triunfos diplomáticos de estas nuevas naciones fueran, hasta fecha muy recierite, bastante sorprendentes. Al comienzo de la década del 60, especialmente después de la masacre de Sharpeville en 1960, las Naciones Unidas, presionadas por los estados africanos, aprobaron resoluciones cada vez más numerosas y urgentes, recomendando una acción internacional en contra de Sudáfrica. En 1962, la Asamblea Gencral recomencló un e:abargo de armas y un boycote comercial, lo que fuc reiterado al año siguiente por el Conscjo de Seguridad. Hacia fines de 1963, no menos de 46 naciones habian intormado al Sectetario Ceneral que habian suscrito la resolución que solicitaba la ruptura de relaciones diplomáticas $;$ comerciales, y luego, 21 paises más anunciaron 
públicamente la mi:ma deteranación. Hacia 1940. el cmbargo le arms se habfa hecho casi univeral y las presiones africanas habian conxiguidu excluit a Sudáfrica de organiziciones internacionales inportantes, tales como la Organización Intcrnacional del Trabajo (orr), la Comisión Económica para Africa de las Niaciones Unidas y la Comunidad Britinica de Niaciones.

Resoluciones similarmente hostiles fueron abrumadoramente aprobadas por la Asamblea General, condenando la politica portuguesa en Angola Y Mozambique y criticandio a Gran Bretaña por su actuación en Rhodesia del Sur. Hay que añadir a lo anterior, que tocla la cuestión de las sanciones económicas reforzadas internacionalmente contra Sudáfrica, fue propucsta por un comité de las Naciones Unidas y revisada a muy alto nivel por los gobiernos de Gran Bretaña y los Estados Unidos.

De este modo, hasta hace sólo dos años, parecía cxistir una genuina posibilidad de que la supremacía blanca en el Africa del Sur no pudiera abrigar la esperanza de mantenerse por mís tiempo. Temiendo tanto ataques externos como revueltas internas, los sudafricanos blancos se vieron obligados a incrementar sus fuerzas armadas hasta un grado sin precedentes. El presupuesto para la defensa en $1965 / 66$ fue de $\$ 322.000 .000$-cinco veces superior al de 1959/60. X, como resultado del embargo de armas impuesto por las Naciones Unidas, el presupuesto actual incluye la suma de $\$ 70.000 .000$ para la manufactura doméstica de armamento moderno bajo licencia extranjera ${ }^{8}$.

Los demás países de Africa del Sur, bajo dominio blanco, se hallaban igualmente inquietos. Hace dos años, los colonos blancos de Rhodesia del Sur se vieron enfrentados con un gobierno laborista en Gran Bretaña, marcadamente más hostil hacia ellos que el gobierno conservador precedente. Los inversionistas comenzaban a perder confianza, iniciándose la fuga de capitales. Muchos rhodesianos blancos iniciaron un éxodo hacia Sudáfrica. También el Africa Sudoccidental constituía un problema para los partidarios de la supremacía blanca, pues existía la posibilidad de que el caso planteado ante la Corte Internacional de Justicia pudiera resolverse en contra de Sudáfrica y, por consiguiente, reforzar aún más el clamor por una acción internacional. Con la inminente independencia que el Alto Comisionado Británico habria de otorgar a los territorios de Bechunalandia, Swazilandia y Basutolandia, muchos estaban convencidos de que debido a su crucial posición estratégica, dichos territorios podrian ser utilizados como trampolines para un asalto contra Sudáfrica. Se temía también que estos paises sirvieran como "vitrinas" de la democracia, a las que los sudafricanos pudieran asomarse con envidia o vergūenza -según fueran ellos negros o blancos'. Además, en Angola y Mozambique, la actividad guerrillera ocupaba a muchas unidacles del cjército por-

\footnotetext{
Ver mi trabajo para la Confetencia Internacional sobre el Africa Sudoceidental, en Oxford, en marzo de 1956 . South West Africe: the defense position.

- Reseña de Neville Rubin en Venlure, junio de 1965 , sobre el libro de Jack Halpern, Sosth Africa's Hustazes, Penguin, 1966.
} 
tugues, lo que hacia presentir que muy pronto esta carga colonial, resultaria excesivamente pesada para Porugal.

Una causa más de ansiedad para los colonos blancos fue la formación, en 1969, de la Organización de la Unidad Africana, inspirada en algunas de las doctrinas panafricanistas de Kwame Nkrumah. En la primera reunión en la cumbre de Addis Abeba, en mayo de 1963, se constituyó un Comité de Coordinación de nueve estados ${ }^{10}$ para canalizar la ayudara los diversos movimientos de liberación de África del Sur, y al año siguiente, en una reunión en El Cairo, los aportes al Fondo de la Libertad administrado por dicho Comité, se hicieron obligatorios para todos los estados miembros, a la vez que fue creado un departamento especial, destinado exclusivamente a ocuparse de los asuntos sudafricanos.

Sin embargo, durante los últimos dos años, hemos visto que evidentemente el poder del nacionalismo africano, tan efectivo en lograr independizarse del yugo colonial, se ha convertido hoy por hoy, en una fantasia. Anteriormente se insinuó cuáles eran las razones para ello: el fin de la guerra fria y la creencia existente en el Occidente de que la balanza donde se equilibran los estados progresistas y conservadores de África, se inclina marcadamente hacia estos últimos y que, por consiguiente, la tan natural aspiración africana de liberar al África del Sur, no se justifica ni por necesidades estratégicas ni politicas.

Pero las consecuencias de los sorprendentes cambios ocurridos deben mirarse más de cerca. Como ja se ha dicho anteriormente, hasta hace dos años, las perspectivas eran optimistas para todos aquellos que deseaban la pronta caida de la supremacia blanca. Hoy dia sucede lo contrario. La fecha que más adecuadamente sirve para marcar este cambio es el 11 de noviembre de 1965 -dia en que el Sr. Smith, ilegalmente, declaró a Rhodesia independiente de Gran Bretaña. Hasta aquj, este hecho ha constituido una victoria de proporciones para su camarilla racista. Ningún signo aparente de debilitamiento ha podido observarse, ni en la resolución del propio Sr. Smith en proseguir por el camino presente, ni en el apoyo que le otorga Ia minoria blanca de Rhodesia. El absurdo optimismo del Primer Ministro británico, quien en enero de 1966 anunciara que la caida de Rhodesia era más bien cuestión de semanas que de meses, deberia compararse a la sobria declaración hecha por los primeros miniștros de la Comunidad Británica en septiembre del mismo año, quienes cxpresaron en sù comunicado que, si bien las actuales "sanciones económicas habian, sin duda, debilitado la economia de Rhodesia, resultaba improbable que al nivel presente logramn obtener los objetivos politicos descados, por lo menos en un periodo razonable"11. Si Smith no cedia, Gran Bretaña habia prometido pedir a las Xlaciones Unidas que impusiera sanciones obligatorias contm Rhodesia. Pero como ni las Naciones L'nidas ni Gran Bretaña

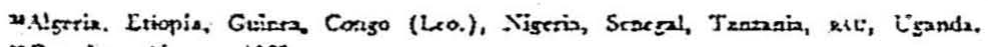

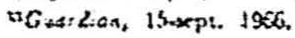


están en posición de póder vigilar las fronteras de Rhodesia, las sanciones "obligatorias" resultaran tan inoperanes como las actuales. Pondrán impedimentos al mercado de Rhodesia pero no lo paralizarán). $Y$ como lo cierto es que las sanciones existences ya le han costado a Gran Bretaña más de \$ 300.000 .000 , resulta dificil imaginar que esta confrontación económica pueda mantenerse indefinidamente. Desde todo punto de vista los blancos en Rho. desia ya han gar ido. Otro factor que confirma lo anterior es, la probabilidad de que el nucvo Primer Ministro sudafricano, Vorster, otorgue a Rhodesia un amplio apojo tanto diplomático y' comercial como militar, si fuera necesario - cosa que su más diplomático antecesor nunca se atrevió a hacer.

Otros cambios también han contribuido a reforzar la posición blanca. Botswana y Lesotho son ahora independientes, pero cstán muy lejos de cons. tituir una amenaza o una perturbación para Sudáfrica por sus diligentes planes de desarrollo para los africanos, pues Gran Bretaña les otorgó la independencia sin salvaguardarlos adecuadamente, y dependen integramente de la buena voluntad sudafricana para poder sobrevivir tanto política como económicamente. El primer ministro de Lesotho, visitó al Dr. Verwoerd justo antes de su muerte, siendo el primer dirigente africano que haya entablado conversaciones con los sudafricanos blancos y ha declarado con frecuencia que Lesotho no apoyará sanción alguna en contra de Sudáfrica13.

Del mismo modo, el fallo de la Corte Internacional de Justicia respecto del Africa Sudoccidental, si bien no tan desastroso como una decisión directamente favorable a Sudáfrica constituye, a pesar de todo, una grave derrota que sólo sirve para reforzar la determinación del gobierno sudafricano de aferrarse a un territorio de tan vital importancia para él. La base militar ilegal actualmente en construcción en Caprivi Strip, será con toda seguridad acelerada al máximo para impedir asi que Zambia y Botswana logren tomar altruna acción significativa contra aquel Mandato.

Pero los cambios verdaderos se han operado en los estados independientes africanos mismos, y en el completo fracaso de sus ofensivas diplomáticas. Es en el caso de Rhodesia donde su debilidad se ha hecho más patente. En las Naciones Unidas han sido dejados fuera de combate y permancntemente desarticulados por el Primer Ministro británico. La Organización para la Unidad Africana se encuentra prácticamente liquidada. Pocos estados, actualmente, escuchan sus recomendaciones o hacen caso de sus decisiones. El 3 de diciembre de 1965 la ova votó unánimemente en favor de una declaración de guerra a Rhodesia y una ruptura de relaciones diplomiticas con Gran Bretaña si Smith no era derrotado hacia el 15 de diciembre cle ese mismo año. Solamente diez paises obedecieron la orden de ruptura de relaciones y ninguno declaró la guerra. Cuando en las Naciones Unidas, durante un discurso del Sr. Wilson el 16 de diciembre, los africanos decidieron abandonar todos la sala por lo

2 Guatdion, editorial, 1t-sept. 1966.

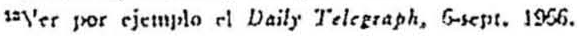


menos nuese paises alricanos se negran a hacerlo. Luego, en febrero de $190 \tilde{j}$. durante la conferencia de ta ova en Adluis Abeha. justo después del golpe que derroci) al Presidente Nikrumah, los escasos paises radicales se vieron obligados a salir de la sala en señal de protesta. La conferencia rechazó una proposición argelina para "intensificar la organización... de los nacionalistas en Rhodesia con miras a iniciar la acción armada dentro del país y de reorganizar la "Unión del Pueblo Africano Zimbabwe" como único movimiento de liberación dentro de Rhodesia"14. Esto fue rechazacio en favor de una resolución mís moderada. El fracaso de la ous se debe, en parte, a la antigua fistura existente entre Casablanca y Brazzaville. Surge también del sentimiento que los africanos tienen de su propia debilidad. Este punto fue abordado con franqueza por el Primer Ministro de Singapore, Lec Kuan I'ew, quien declaró después de la reciente conferencia de la Comunidad Británica en Lonclres: "Lo que me desalentó fue la sensación de impotencia que pude advertir en un gran número de nuestros colcgas africanos por carecer del suficiente poder militar para derrotar a 200.000 blancos. . ninguno de ellos, independientemente o aliados entre si, son capaces de enfrentarse a 200.000 blancos en Rhodesia"15.

Pero lo más grave ha sido el resultado de los golpes militares que han transformado por completo el panorama africano. El ejército ha intervenido, de hecho, diez veces en los últimos cuatro años para derrocar gobiernos africanos y siete veces durante $1966^{16}$. Si se recuerda que estas cifras incluyen a países tan importantes y significativos como Argelia, el Congo, Nigeria y Ghana, resulta fácil descubrir por qué en el presente, Africa parece carecer de dirección. Casi sin excepción, los cambios de regímenes han conducido a un mayor acercamiento al Occidente. Las frecuentes expulsiones de técnicos y diplomáticos chinos y europeos orientales han simbolizado la transformación del viejo concepto de neutralidad entre Oriente y Occidente.

Dentro del contexto de la lucha por la liberación del Africa del Sur de la supremacía blanca, esta evolución resulta profundamente inquietante. Pues aunque el Occidente $-y$ me refiero principalmente a los Estados Unidos, Gran Bretaña y Francia- aparece tan vociferante en su desaprobación moral de la supremacía blanca como los estados comunistas, sus compromisos económicos en el Africa del Sur son de tal magnitud que no puede encarar con tranquilidad, cualquier acción que constituya una amenaza hacia estos intereses 17 . 1965.

"Ver de Nora Mfckeon, The African States and the ost, International Affairs, julio,

${ }^{15}$ Durante uns conferencia de prensa en Singapur, Cuardian, 24 sept. 1966.

29Togo, Congo (Brazaville), Dahotne), Gabon, Argelia, Congo (Le toldville), Rep. del Árica Central, Ato Volta, Nigeria, Ghana.

i:Este problema cstá tratado ampliamente en ol libro de Dennis Austin: Brilcin and South 1/rica, Oxford University P'rets, 1966. Lamentablemente recibi un ejemplar después de haber publicado este artículo. Nuestras conclissiones son muy similares. 
Tampoco puede el occidente financiar ficintente el costo que tal aecion semandaria. Derribar el regimen blanco de Rhoulesia por la fuerza. que es. como to han reconocicio los dirigentes de la Comunidad Britínica de Na. ciones "la única manera segurn" de conseguirlo significarta el despliegue de una fuerza de unos 90.000 hombres. Transporte militar aéreo, tanto uritánico como norteamericano, tendria que ser emplendo, probablemente, en una escala considerable 1 .

Las sancioncs económicas con Sucláfrica misma, según cálculos preparados en 1964 basados en la experiencia norceamericana del bloqueo a Cuba, podrían costar sobre $\$ 150.000 .000$ mensuales $^{10}$. El año pasaclo, el delegado británico ante las Naciones Unidas declaró: "debemos aceptar èl hecho de que una campaña intensiva de sanciones económicas respalclaclas por un bloqueo requeririan recursos superiores a la capacidad presente de las Naciones Unidas". Y con el incremento de la capacidad defensiva de Sucláfrica resulta dudoso que incluso las sanciones militares logren alguna efectividad o sean toleradas por la opinión occidental. Esa nación pronto tendrá gases venenosos para uso militar a su disposición y dentro de una década puede llegar a poseer una capacidad atómica considerable ${ }^{20}$. Considerando todos estos factores, el occidente actuaría contra Sudáfrica solamente bajo presiones extremas. Y estas presiones, por el momento han clesaparecido.

Nos encontramos asi ante un problema que, como fue señalado anteriormente, ha desconcertado al mundo durante los últimos veinte años. La clave se encuentra en los principales poderes occidentales y en sus aliados, pues son ellos los mayores auspiciadores del régimen sudafricano. Pero, de no ocurrir cambios imprevisibles, transcurrirán por lo menos otros veinte años antes de que la comunidad internacional llegue a ser testigo de que se haga justicia bacia la mayoría negra que puebla las regiones meridionales del continente africano. Las presiones ejercidas por los africanos y los paises comunistas sobre el occidente son demasiado débiles y sólo reforzándolas es como podría producirse un cambio. Esto significaría, en primer lugar, que los paises del mundo comunista tomaran parte activa en los problemas africanos, haciendo revivir asi el temor al comunismo de hace algunos años; y en segundo lugar, el mejoramiento de las actuales divisiones existentes entre el Africa independiente y el movimiento de liberación del sur. Ninguna de estas dos posibilidades parece probable en la actualidad. Hay quienes argumentan, utilizando un clásico análisis de clases, que el desarrollo industrial y el progreso económico acarrearan automáticamente una mejoria en las relaciones entre la clase trabajadora negra y la burguesia blanca. Pero este argumento, fuera de otros defectos, desconoce el hich q de que un choque racial se ha superpuesto sobre un conflicto de clases tradicional. Probablemente la mayor esperanza nace de

2s: Neville Brown, Venture, enero, 1966.

aConferencia Internacional sobre Sanciones.

xVer mi trabajo, swa, the defoxse position. 
los propios movimientos de libención africanos. Se confirmarla entonces la tesis enunciada hace algunos años por el dirigente negrö sudafricano Robert Sobukwe, que únicamente el africano mismo será capaz de conquistar su propia saliación politica. Pero, por el momento, la oposición blanca es demasiado [uerte. Unicamente en Guinea portuguesa -muy alejada del Africa del Sur y de tan poca importancia cconómica- puede verse señas de verdadero éxito21. El ejército en Angola y Mozambique aún controla la situación, y la policia en Sudífrica está singularmente bien equipada y bien informada. Hace algunos meses, el Parlamento Sudafricano aprobó una nueva ley que permite arrestar y detener a cualquier persona sospechosa de sabotaje o de haber tenido entrenamiento terrorista en el extranjero. Si algún día, los ejércitos de liberación del Africa del Sur se demuestran efectivos, será la tarea de aquellos que se encuentran fuera de Africa y que comparten las objeciones morales hacia la cloctrina de la supremacia blanca, no solamente el impedir que sus gobiernos comercien con Sudáfrica sino además, que intervengan militarmente del lado de los blancos22.

No faltarán quienes se regocijen ante la perspectiva, anotada aqui, de una prolongación casi indefinida de la supremacia blanca en Africa del Sur. Pero, a manera de conclusión, vale la pena recalcar que la permanencia en el poder de un grupo de racistas blancos constituye no sólo una afrenta para los valores morales que las democracias, tan frecuentemente en el pasado, lucharon por mantener, sino además, una amenaza para aquel sistema internacional que tan sacrificadamente se ha venido construyendo desde el término de la Primera Gucra Mfundial. Ninguna organización, como las Naciones Unidas, puede sobrevivir si sus decisiones y recomendaciones son constantemente despreciadas. $Y$ de este modo, a nuestro entender, asi como los acontecimientos del mundo fuera de Africa son; en parte, responsables del actual fracaso de los estados africanos en derrotar a la supremacia blanca; tampoco cabe duda que la prolongación del dominio blanco interno, forzosamente deberá traer repercusiones indeseables en el mundo exterior. Los cambios sucederán en Sudáfrica, como en Vietnam y en otras regiones en conflicto, cuando las actitudes de las grandes potencias obedezcan a un sentido moral

$=\mathrm{Ver}_{\mathrm{e}}$ el importante artículo de Amilear Cabral, The Struggle in Guinca, en International Socialist Journal, agosto, 1964.

=La posición Irancesa resulta particularmente curiess en ate conflicto. Francia parece haber nerado el principio de no intervención al nivel de un dogina de fe. Así como constituye un efror la intervención de los Estados Unidos en Vietram o en Sarto Domingo, también lo es cualquier intervención (incluso de las Naciones Linidas) en cualąuicr lugar. En consecuencia, Y con aparente légica, Francia se epone a toda resolución adoptada por las Naciones Unidas que tienda a facilitar la interiención de has Naciones Ĺnidas en el Africa del Sur. Animada pur tan paderose ereencia en les bondades de ta po-interención, francia $x$ da la libertad de coarereiar abiertamente con Sudhlsiea llegando hasta el extremo de apjertrecharla de arma-

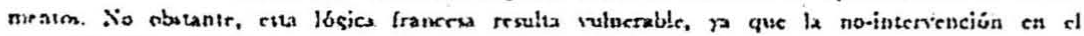
cass del Afrisa del Sur ia contro ta Carta de las lixciones Ĺnidas y contro los derechios huE-2:00s londanentalos. 
Rishara Goft $/$ El Sur de Africa $y$ el fin de is suerra tsia

más que a un errónco sentido de autointerés a corto plazo. Lo anterior solamente será posible cuando sea más amplia la participación en los procesos en que se forman las decisiones.

In la actualidad, son muchos los paises que se permiten vivir en un falso liberalismo, que a la vez que condena los males del apartheid, alienta la creencia de que algo puede hacerse al respecto. El hecho cierto es que, dada la situación preser. e no existe la perspectiva inmediata de que la supremacia blanca pueda ser derrocada por los medios contemplados hasta aqui. Sólo cuando este hecho sea admitido podrán surgir nuevos métodos para enfrentar este problema. 